\title{
SPOLM2019
}

XIX SIMPÓSIO DE PESQUISA OPERACIONAL ELOGÍSTICA DA MARINHA

\section{Sistema de Apoio à Decisão “JS-ELECTRE” desenvolvido em JavaScript: uma plataforma on line com grafos dinâmicos}

\author{
Valdecir da Silva Pereira \\ Instituto Militar de Engenharia - IME \\ Praça General Tibúrcio, 80, 22290-270, Praia Vermelha Rio de Janeiro, RJ, Brasil \\ vvaldecirr@hotmail.com \\ Marcos dos Santos \\ Instituto Militar de Engenharia - IME \\ Praça General Tibúrcio, 80, 22290-270, Praia Vermelha Rio de Janeiro, RJ, Brasil \\ marcosdossantos_doutorado_uff@yahoo.com.br
}

\begin{abstract}
RESUMO
Mesmo nos tempos mais remotos, tomar decisões nunca foi uma tarefa fácil, principalmente em problemas maus estruturados e com inúmeros critérios conflitantes. Assim, torna-se necessário utilizar métodos que sejam capazes de estruturar problemas levando em consideração as múltiplas perspectivas das partes interessadas. O que se busca não é eliminar a subjetividade do processo decisório, mas sim uma maneira de estruturar de maneira racional essa subjetividade natural da psiché humana. Dentro deste escopo, este trabalho tem o propósito de apresentar um Sistema de Apoio à decisão que calcula os resultados do método ELECTRE de maneira simples e intuitiva, considerando que se trata de um dos métodos consagrados da Escola Francesa mais utilizados do mundo. Desenvolvido pelos pesquisadores do Programa de Pós-graduação em Sistemas e Computação do Instituto Militar de Engenharia (IME), o sistema foi implementado em JavaScript, podendo ser acessado pelo endereço https://vvaldecirr.github.io/JSElectre/index.html. Com este estudo, espera-se trazer uma significativa contribuição para a sociedade, principalmente para a comunidade acadêmica, uma vez que a ferramenta apresentada funciona totalmente online em qualquer sistema operacional e em qualquer navegador, não sendo necessário baixar ou instalar um software específico para a utilização do método ELECTRE. Como Inovação, o JS-ELECTRE apresenta uma interface gráfica que mostra as alternativas dominadas e as alternativas dominantes a partir de Grafos dinâmicos, cujos vértices e arcos se reorganizam de acordo com os valores atribuídos aos graus de concordância e discordância.
\end{abstract}

Palavra-chave: Método ELECTRE; Apoio Multicritério à Decisão (AMD); JS-ELECTRE. 


\begin{abstract}
Even in the earliest times, making decisions has never been an easy task, especially in poorly structured problems with numerous conflicting criteria. Thus, it is necessary to use methods that are able to structure problems taking into account the multiple perspectives of stakeholders. The aim is not to eliminate the subjectivity of the decision making process, but rather a way of rationally structuring this natural subjectivity of the human psyche. Within this scope, this paper aims to present a Decision Support System that calculates the results of the ELECTRE method in a simple and intuitive way, considering that it is one of the most widely used French School methods in the world. Developed by researchers from the Postgraduate Program in Systems and Computing of the Military Institute of Engineering (IME), the system was implemented in JavaScript, which can be accessed at https://vvaldecirr.github.io/JSElectre/index.html. With this study, it is expected to make a significant contribution to society, especially to the academic community, as the presented tool works fully online in any operating system and browser, and it is not necessary to download or install software specifically for the use of the ELECTRE method. As an innovation, JS-ELECTRE features a graphical interface that shows dominated and dominant alternatives from dynamic graphs, whose vertices and arcs rearrange according to the values assigned to the degrees of agreement and disagreement.
\end{abstract}

Keywords: ELECTRE Method; Multicriteria Decision Aid (MCDA); JS-ELECTRE.

\title{
Como Citar:
}

PEREIRA, Valdecir da Silva; SANTOS, Marcos dos. Sistema de Apoio à Decisão "JSELECTRE" desenvolvido em JavaScript: uma plataforma on line com grafos dinâmicos. In: SIMPÓSIO DE PESQUISA OPERACIONAL E LOGÍSTICA DA MARINHA, 19., 2019, Rio de Janeiro, RJ. Anais [...]. Rio de Janeiro: Centro de Análises de Sistemas Navais, 2019.

\section{INTRODUÇÃO}

Segundo [8], a realidade é de fato multidimensional, assim, o que está em causa é uma representação de aspectos relevantes dessa realidade, e nunca a própria realidade.

Este fato, por si só, já justifica a necessidade da exploração, cada vez mais eficaz, dos recursos computacionais com desenvolvimento de ferramentas eficientes no auxílio à tomada de decisão. Conforme afirmam [9], a velocidade das mudanças, dos elevados volumes financeiros investidos e do aumento do nível de incerteza, tornam a tomada de decisão mais difícil e se faz necessário usar ferramentas cada vez mais sofisticadas para tratar o problema.

\section{FUNDAMENTAÇÃO TEÓRICA}

\subsection{Multi Criteria Decision MaKing (MCDM)}

MCDM, ou Tomada de Decisão Multicritério, é uma técnica baseada na existência de diversas alternativas onde os processos de tomada de decisão levam em consideração vários critérios atrelados ao sistema. 
Conforme [1], há uma necessidade de que implementações de métodos MCDS em sistemas de recomendação ainda sejam exploradas sistematicamente. Sistemas de Recomendação conseguem elucidar alguns sistemas de contribuição que englobam vários métodos MCDM. Tais sistemas possuem aplicações em diversificados domínios. Por outro lado, com uma análise mais compreensiva, torna-se mais fácil o entendimento e o desenvolvimento de um sistema que possa identificar dimensões capazes de distinguir, explicar e categorizar sistemas de recomendação multicritério, baseados em uma taxonomia existente e categorização utilizada em análises e classificações de tomadas sobre exemplos de decisões de usuários.

MCDM é uma teoria de tomada de decisão que leva em consideração um limitado conjunto de opções alternativas contra vários critérios, sendo assim, o problema pode ser formulado da seguinte forma:

Supondo que existem $\mathrm{M}$ critérios e $\mathrm{N}$ alternativas, deve-se escolher alguma ou um conjunto de alternativas a qual satisfaça ao máximo os critérios. Este problema MCDM pode ser apresentado na matriz de decisão da Tabela 1.

Tabela 1: Matriz de Decisão. Fonte: Marlinda et al. (2017)

\begin{tabular}{|c|c|c|c|c|c|}
\hline \multicolumn{6}{|c|}{ Criteria } \\
\hline & $C_{1}$ & $C_{2}$ & $C_{r}$ & ... & $C_{N}$ \\
\hline$\underline{\text { Alternative }}$ & $W_{l}$ & $W_{2}$ & $W_{3}$ & $\ldots$ & $W_{N}$ \\
\hline$A_{1}$ & $a_{11}$ & $a_{12}$ & $a_{13}$ & $\cdots$ & $a_{l N}$ \\
\hline$A_{2}$ & $a_{21}$ & $a_{22}$ & $a_{23}$ & ... & $a_{2 N}$ \\
\hline$A_{3}$ & $a_{31}$ & $a_{32}$ & $a_{33}$ & $\cdots$ & $a_{3 N}$ \\
\hline . & . & - & . & $\cdots$ & . \\
\hline$\cdot$ & $\cdot$ & $\cdot$ & $\cdot$ & $\cdots$ & $\cdot$ \\
\hline$\cdot$ & $\cdot$ & $\cdot$ & $\cdot$ & $\cdots$ & $\cdot$ \\
\hline$A_{1}$ & $A_{M I}$ & $A_{M 2}$ & $A_{M 3}$ & ... & $A_{M N}$ \\
\hline
\end{tabular}

A matriz de decisão é um elemento de tamanho $\mathrm{M} x \mathrm{~N}$ onde o elemento aij indica a performance da alternativa Ai quando confrontada com o critério Cj (para i = 1, 2, 3, ... M e J = 1, 2, 3, .., N).

\subsection{ELIMINATION ET CHOIX TRAdUISANT LA RÉALITÉ(ELECTRE)}

O primeiro método ELECTRE foi apresentado por Benayoun, Roy, Sussman em 1966, mas a publicação do primeiro artigo somente ocorreu em 1968, quando Roy descreveu o método em detalhes, sendo após renomeado para ELECTRE I. O nome ELECTRE Iv (v de veto) é utilizado algumas vezes quando os limiares de veto são levados em conta, mas não é considerado um nome oficial. Diversos outros métodos ELECTRE foram desenvolvidos durante as duas décadas seguintes. Cada versão do ELECTRE difere operacionalmente entre si, mas eles também diferem com relação aos tipos de problemas onde eles podem ser aplicados. [2] 


\subsection{ELECTRE I}

O conceito básico neste método consiste na ênfase da análise das relações de dominância das alternativas entre si. O objetivo é encontrar um subconjunto de alternativas dominantes as quais não pertencem ao outro subconjunto de alternativas sobreclassificadas pelo primeiro. O que permite que estes subconjuntos sejam definidos, é a utilização dos índices de concordância, que mensura a relação de vantagem de cada alternativa em confrontamento com as demais, e o índice de discordância, medindo por sua vez a desvantagem relativa. Os métodos ELECTRE consideram os índices peso como uma medida de importância que cada critério recebe do decisor, logo, estes índices são utilizados para se computar os coeficientes de concordância e discordância.

A concordância testa a afirmação de que um subconjunto significativo de critérios concorda que a alternativa a sobreclassifica (é pelo menos tão boa quanto) b.

A discordância testa a afirmação de que o restante dos critérios discordam fortemente de que a alternativa a sobreclassifica $b$.

A notação básica do método ELECTRE I assume:

- $a_{i}=$ Alternativa $i$

- $g_{j}=$ Critério $j$;

- $g_{j}\left(a_{i}\right)=$ Desempenho da alternativa i em relação ao critério j;

- $W_{j}=$ Peso do critério $j$;

- $\quad c_{l k}\left(a_{l}, a_{k}\right)=$ Grau de concordância entre as alternativas $l$ e $k$;

- $\quad d_{l k}\left(a_{l}, a_{k}\right)=$ Grau de discordância entre as alternativas $l$ e $k$;

- $\quad S_{l k}\left(a_{l}, a_{k}\right)=$ Grau de dominância (credibilidade) de uma alternativa;

- $\quad p=$ Valor de corte para a concordância. Normalmente $0.5 \leq p \leq 1$;

- $q=$ Valor de corte para a discordância. Normalmente $0 \leq q \leq 0.5$;

A Matriz de Performance, Tabela 2, é utilizada para o preenchimento dos valores e posterior análise da necessidade de normalização.

Tabela 2: Matriz de Performance. Fonte: Pereira (2017)

\begin{tabular}{|c|c|c|c|c|c|}
\hline & $g_{1}$ & $g_{2}$ & $g_{3}$ & $g_{4}$ & g5 \\
\hline$a_{1}$ & $g_{1}\left(a_{1}\right)$ & $g_{2}\left(a_{1}\right)$ & $g_{3}\left(a_{1}\right)$ & $g_{4}\left(a_{1}\right)$ & $g_{5}\left(a_{1}\right)$ \\
\hline$a_{2}$ & $g_{1}\left(a_{2}\right)$ & $g_{2}\left(a_{2}\right)$ & $g_{3}\left(a_{2}\right)$ & $g_{4}\left(a_{2}\right)$ & $g_{5}\left(a_{2}\right)$ \\
\hline$a_{3}$ & $g_{1}\left(a_{3}\right)$ & $g_{2}\left(a_{3}\right)$ & $g_{3}\left(a_{3}\right)$ & $g_{4}\left(a_{3}\right)$ & $g_{5}\left(a_{3}\right)$ \\
\hline$a_{4}$ & $g_{1}\left(a_{4}\right)$ & $g_{2}\left(a_{4}\right)$ & $g_{3}\left(a_{4}\right)$ & $g_{4}\left(a_{4}\right)$ & $g_{5}\left(a_{4}\right)$ \\
\hline as & $g_{1}\left(a_{5}\right)$ & $g_{2}\left(a_{5}\right)$ & $g_{3}\left(a_{5}\right)$ & $g_{4}\left(a_{5}\right)$ & $g_{5}\left(a_{5}\right)$ \\
\hline Pesos & $W_{1}$ & $W_{2}$ & $W_{3}$ & $W_{4}$ & $W_{5}$ \\
\hline
\end{tabular}

Para a matriz de concordância, Tabela 3, normaliza-se a matriz de performance, depois soma-se os pesos dos critérios (devem ser normalizados se o seu somatório é diferente de 1 nos quais a alternativa $\boldsymbol{a}_{\boldsymbol{l}}$ supera à alternativa $\boldsymbol{a}_{\boldsymbol{k}}$, quer dizer que, $\boldsymbol{a}_{i j} \geq \boldsymbol{a}_{k j}$. 
Concordância da alternativa $a_{l}$ em relação a $a_{k}$ :

$$
c_{l k}=\frac{\sum_{j: a_{l} s_{j} a_{k}} w_{j}}{\sum_{j} w_{j}}
$$

Tabela 3: Construção da matriz de concordância. Fonte: Pereira (2017)

\begin{tabular}{|c|c|c|c|c|c|}
\hline & $a_{1}$ & $a_{2}$ & $a_{3}$ & $a_{4}$ & $a 5$ \\
\hline$a_{1}$ & - & $c_{12}$ & $c_{13}$ & $c_{14}$ & $c_{15}$ \\
\hline$a_{2}$ & $c_{21}$ & - & $c_{23}$ & $c_{24}$ & $c_{25}$ \\
\hline$a_{3}$ & $c_{31}$ & $c_{32}$ & - & $c_{34}$ & $c_{35}$ \\
\hline$a_{4}$ & $c_{41}$ & $c_{42}$ & $c_{43}$ & - & $c_{45}$ \\
\hline$a 5$ & $c_{51}$ & $c_{52}$ & $c_{53}$ & $c_{54}$ & - \\
\hline
\end{tabular}

$$
d_{l k}=\left\{\begin{array}{c}
0 \text { se } a_{l j} \geq a_{k j}, \mid \forall_{j} \\
\frac{\text { Máximo positivo }\left(a_{k j}-a_{l j}\right)}{\delta}
\end{array}\right.
$$

$\delta=\max \{$ maior avaliação $j-$ menor avaliação $j\}$

\begin{tabular}{|c|c|c|c|c|c|}
\hline & $a_{1}$ & $a_{2}$ & $a_{3}$ & $a_{4}$ & $a_{5}$ \\
\hline$a_{1}$ & - & $d_{12}$ & $d_{13}$ & $d_{14}$ & $d_{15}$ \\
\hline$a_{2}$ & $d_{21}$ & - & $d_{23}$ & $d_{24}$ & $d_{25}$ \\
\hline$a_{3}$ & $d_{31}$ & $d_{32}$ & - & $d_{34}$ & $d_{35}$ \\
\hline$a_{4}$ & $d_{41}$ & $d_{42}$ & $d_{43}$ & - & $d_{45}$ \\
\hline$a 5$ & $d_{51}$ & $d_{52}$ & $d_{53}$ & $d_{54}$ & - \\
\hline
\end{tabular}

Tabela 4: Construção da matriz de discordância. Fonte: Pereira (2017)

Na matriz de dominância, Tabela 5, é realizado o teste de dominância, onde a alternativa $a_{l}$ domina a alternativa $a_{k}$ se e somente se $c_{l k}>p$ e $d_{l k}<q$. 
Tabela 5: Matriz de dominância. Fonte: Pereira (2017)

\begin{tabular}{|c|c|c|c|c|c|}
\hline \multirow{3}{*}{$\boldsymbol{a}_{1}$} & \multicolumn{2}{c}{$\boldsymbol{a}_{1}$} & \multicolumn{2}{c}{$\boldsymbol{a}_{2}$} & \multicolumn{2}{c}{$\boldsymbol{a}_{3}$} & \multicolumn{2}{c|}{$\boldsymbol{a}_{4}$} & $\boldsymbol{a}_{5}$ \\
\cline { 2 - 6 } $\boldsymbol{a}_{2}$ & - & $S_{12}$ & $S_{13}$ & $S_{14}$ & $S_{15}$ \\
\cline { 2 - 6 } $\boldsymbol{a}_{3}$ & $S_{21}$ & - & $S_{23}$ & $S_{24}$ & $S_{25}$ \\
\cline { 2 - 7 } $\boldsymbol{a}_{4}$ & $S_{31}$ & $S_{32}$ & - & $S_{34}$ & $S_{35}$ \\
\cline { 2 - 7 } $\boldsymbol{a}_{5}$ & $S_{41}$ & $S_{42}$ & $S_{43}$ & - & $S_{45}$ \\
\cline { 2 - 7 } & $S_{51}$ & $S_{52}$ & $S_{53}$ & $S_{54}$ & - \\
\cline { 2 - 7 } & & & & & \\
\hline
\end{tabular}

A partir do Teste de Dominância constrói-se o Kernel do problema (partição).

$$
s_{l k}=\left\{\begin{array}{l}
1, \quad \text { se } c_{l k}>p \text { e } d_{l k}<q \\
0, \text { Otherwise }
\end{array}\right.
$$

Partição do conjunto de alternativas em dois subconjuntos:

- K: Núcleo, ou Kernel ou Não-dominadas: Conjunto de alternativas entre as quais não há relação de sobreclassificação. Estas alternativas são incomparáveis ou não-dominadas entre si.

- D: Conjunto, cujas alternativas são sobreclassificadas por pelo menos uma alternativa em K.

As duas afirmações abaixo devem ser verificadas na partição formada:

- Todas as alternativas em K são incomparáveis entre si.

- Toda alternativa em $D$ é sobreposta por, pelo menos, uma alternativa em K .

Observe que o subconjunto $D$ é dominado pelo subconjunto $K$. Nem todas as alternativas em $D$ precisam ser dominadas por todas as alternativas em $K$. Se o grafo não possuir ciclos o Kernel é único, senão existirão múltiplos Kernels ou nenhum. [3] 


\subsection{HYPERTEXT MARKUP LANGUAGE (HTML)}

A abreviação é oriunda de Hypertext Markup Language (Linguagem de Marcação de Hipertexto), ou seja, o HTML não é uma linguagem de programação mas sim uma seleção de marcadores usados para a diagramação para formatação e publicação de conteúdo (texto, imagens, vídeos, áudio etc.) em págidas na web.

O HTML é baseado no conceito de hipertexto, que são conjuntos de elementos ligados por conexões, que podem ser palavras, imagens, vídeos, áudio, documentos etc. que quando conectados, formam uma grande rede de informação. A conexão feita em um hipertexto é algo imprevisto que permite a comunicação de dados, organizando conhecimentos e guardando informações relacionadas.

Entre 1993 e 1995, surgiram novas versões (HTML+, HTML 2.0, HTML 3.0) onde foram propostas diversas mudanças para enriquecer as possibilidades da linguagem, mas, ela ainda não era tratada como padrão. Apenas em 1997, o grupo de trabalho do W3C, trabalhou na versão 3.2, fazendo com que ela fosse tratada como prática comum

Em 2004, foi fundado o WHATWG (Web Hypertext Application Technology Working Group) por desenvolvedores de empresas como Mozilla, Apple e Opera, onde se iniciou o trabalho de escrever a nova versão do HTML, que seria chamado hoje de HTML5.

O HTML5 é a nova versão do HTML4 e um dos seus principais objetivos é facilitar a manipulação dos elementos, possibilitando o desenvolvedor modificar as características dos objetos de forma não intrusiva, fazendo com que isso fique transparente para o usuário final. Para se ter uma ideia disso, diferente das versões anteriores, o HTML5 fornece ferramentas para o CSS e o Javascript fazerem seu trabalho da melhor possível de forma que um web site ou aplicação continue leve e funcional. [4]

\subsection{Cascading Style Sheets (CSs)}

O CSS nada mais é do que uma linguagem de customização de elementos seja ele HTML, javascript, XML, etc. Com o seu uso é possível estilizar praticamente qualquer coisa em um website.

O CSS1 significou um avanço muito importante na hora de construir websites, permitindo que os desenvolvedores pudessem ter muito mais controle sobre os elementos da página, mas mesmo com esse avanço todo, sempre existe uma coisa ou outra que o desenvolvedor sente necessidade de fazer e que essa versão não permitia que ele fizesse.

Com a chegada do CSS2, algumas novidades interessantes foram incorporadas, o que melhorou e muito a qualidade dos web sites desenvolvidos, mas assim como o que aconteceu com o CSS1, após um tempo tornou-se necessário que tivesse algo a mais para ser acrescentado, pois já haviam algumas coisas que a versão 2 já não estava suprindo, foi aí então que surgiu o CSS3.

O código CSS pode ser aplicado diretamente nas tags ou ficar contido dentro das tags <style>. Também é possível criar um link para um documento CSS externo à página a ser estilizada. Desta forma, quando se quiser alterar a aparência das páginas vinculadas a este arquivo CSS, basta modificá-lo em um único arquivo.

Com a variação de atualizações dos navegadores, o suporte ao CSS pode variar. A interpretação dos navegadores pode ser avaliada com o teste Acid2, que se tornou uma forma base de revelar quão eficiente é o suporte de CSS.

O Doctype informado, ou a ausência dele, determina o quirks mode ou o strict mode, modificando o modo como o CSS é interpretado e a página renderizada. [5] 


\subsection{JAVASCRIPT}

Também conhecido como JS, é uma linguagem de programação, front-end, multiparadigma dinâmica, interpretada e fracamente tipificada. Por ser normalmente utilizada em conjunto com HTML e CSS, o JavaScript é uma das três principais tecnologias presentes em páginas na Web pois viabiliza páginas interativas com menor necessidade de recursos tecnológicos exigidos nas linguagens back-eng.

Devido a maioria dos sites usarem JavaScript, todos os principais navegadores possuem um mecanismo JavaScript embarcado dedicado para interpretação dos scripts. Toda vez que uma página da web faz mais do que simplesmente mostrar informação estática, mostrando conteúdo que se atualiza em um intervalo de tempo, mapas interativos ou gráficos 2D/3D animados, etc., provavelmente o JavaScript está envolvido. É a terceira camada do bolo das tecnologias padrões da web, sendo as outras duas HTML e CSS. [6]

\subsection{JQUERY}

jQuery é uma biblioteca de funções em JavaScript que interage com o hipertexto da página web, visando simplificar os scripts interpretados no navegador.

Criada em dezembro de 2006 no BarCamp de Nova York por John Resig e sendo a mais popular das bibliotecas JavaScript no mundo, jQuery é uma biblioteca de código aberto (open source) e possui licença dual, fazendo uso da Licença MIT ou da GNU General Public License versão 2. Sua sintaxe do foi desenvolvida para simplificar a navegação em documentos HTML, a seleção de elementos DOM, criar animações, manipular eventos, desenvolver aplicações AJAX e criação de plugins sobre ela. Permitindo aos desenvolvedores criarem camadas de abstração para interações de baixo nível de modo simplificado em aplicações web de grande complexidade. [7]

A biblioteca Jquery pode ser utilizada para:

- Adicionar efeitos visuais e animações;

- Acessar e manipular o DOM;

- Carregar componentes Ajax;

- $\quad$ Prover interatividade;

- Fazer alteração de conteúdo;

- Simplificar tarefas JavaScript.

\subsection{ARBOR.JS}

Arbor é uma biblioteca de visualização de grafos construída com componentes web e jQuery. Em vez de tentar ser um framework de estrutura muito abrangente, arbor procura prover um eficiente algoritmo de leiaute direcionado à foças e mais abstrações para organizações de grafos além de gerir atualização de tela. Ele permite que a tela seja redesenhada e atualizada pelo desenvolvedor. Isto significa que pode ser utilizado com canvas, SVG, ou mesmo elementos HTML definidamente posicionados; é apropriado para utilização el qualquer abordagem de exibição e necessidade de desempenho.

Como resultado, o código que for escrito com ele poderá focar em coisas que façam o projeto único - os dados do grafo e estilo visual - em vez de perder tempo com a física e matemática necessária para tornar o leiaute possível. 


\section{DESENVOLVIMENTO DA FERRAMENTA}

O intuito de disponibilizar uma ferramenta baseada em ambiente WEB, com o diferencial de ser completamente open source e que facilite a aplicação da metodologia ELECTRE I de uma forma simples e isentando a necessidade de prévio conhecimento técnico do usuário, fomentou o desenvolvimento do software JSElectre 1.0, quando no curso da disciplina de Desenvolvimento de Plataformas Computacionais de Apoio à Tomada de Decisão, do Programa de Mestrado em Sistemas e Computação do Istituto Militar de Engenharia (IME).

Segundo estudo realizado em 2015 pela Universidade de Coimbra [8], por mais apelativa que seja uma metodologia de apoio à decisão, esta dificilmente terá expressão prática se não existir um software que facilite a sua implementação. E a aplicação que mais se aproximou da proposta do JSElectre, foi a ferramente denominada Matrix, disponível em: http://mad.dec.uc.pt/matrix, porém é de código fechado de direito mantido pelos desenvolvedores.

\subsection{MODELAGEM}

Primeiramente foi necessário reproduzir toda a metodologia manualmente em uma planilha eletrônica, conforme exibido na Figura 1, a fim de elucidar completamente a metodologia ELECTRE I para somente após, de uma maneira mais eficiente, elaborar quaisquer estratégias de implementação.

Figura 1: Planilha para estudo do método ELECTRE I. Fonte: Autores (2019)

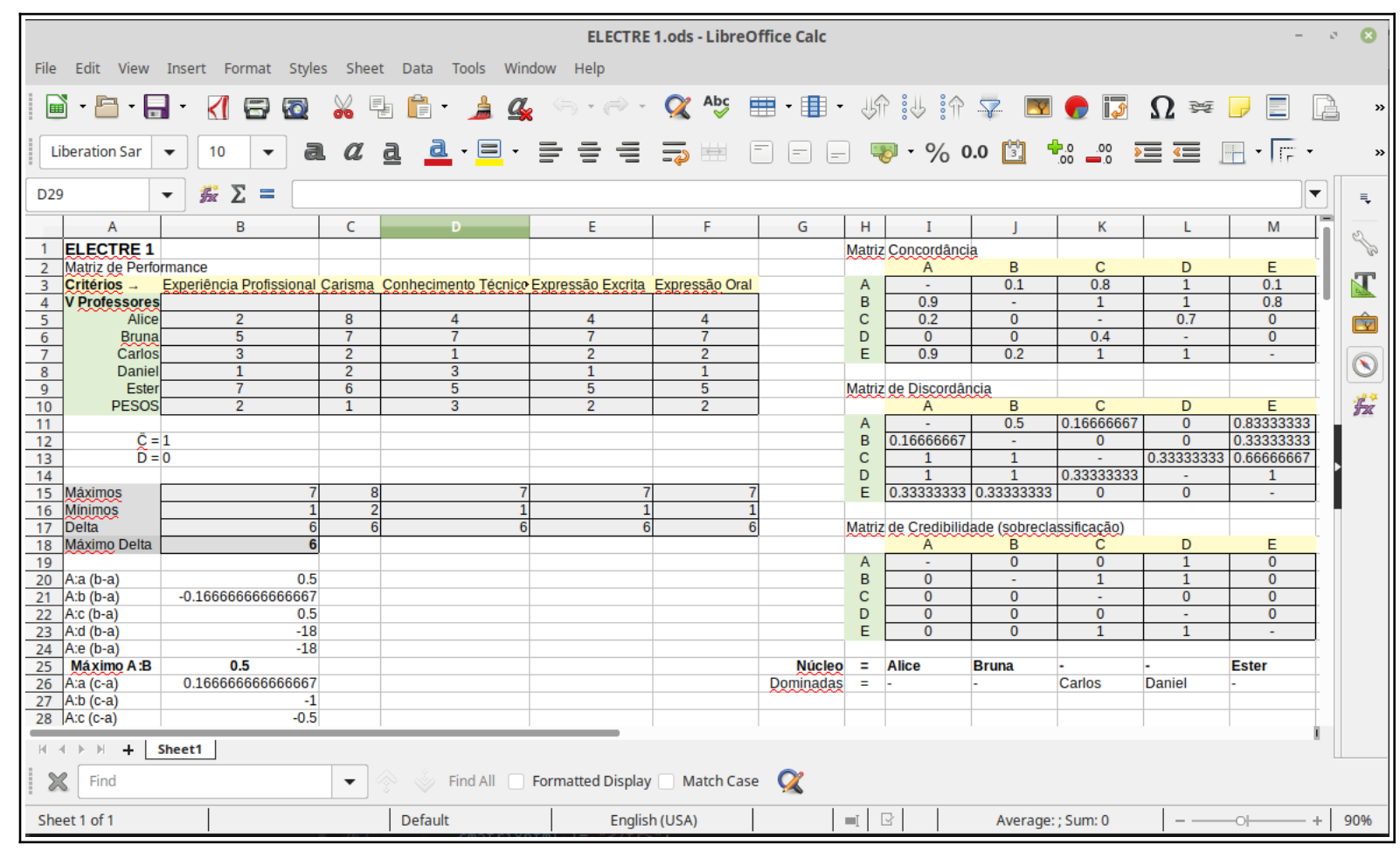

No simples diagrama de casos de uso da Figura 2, estão definidos os papéis e ações de cada ator no sistema em passos bem descritos e suas relações que moldam a estratégia elaborada para a implementação do algoritmo. 
Figura 2: Diagrama de casos de uso. Fonte: Autores (2019)

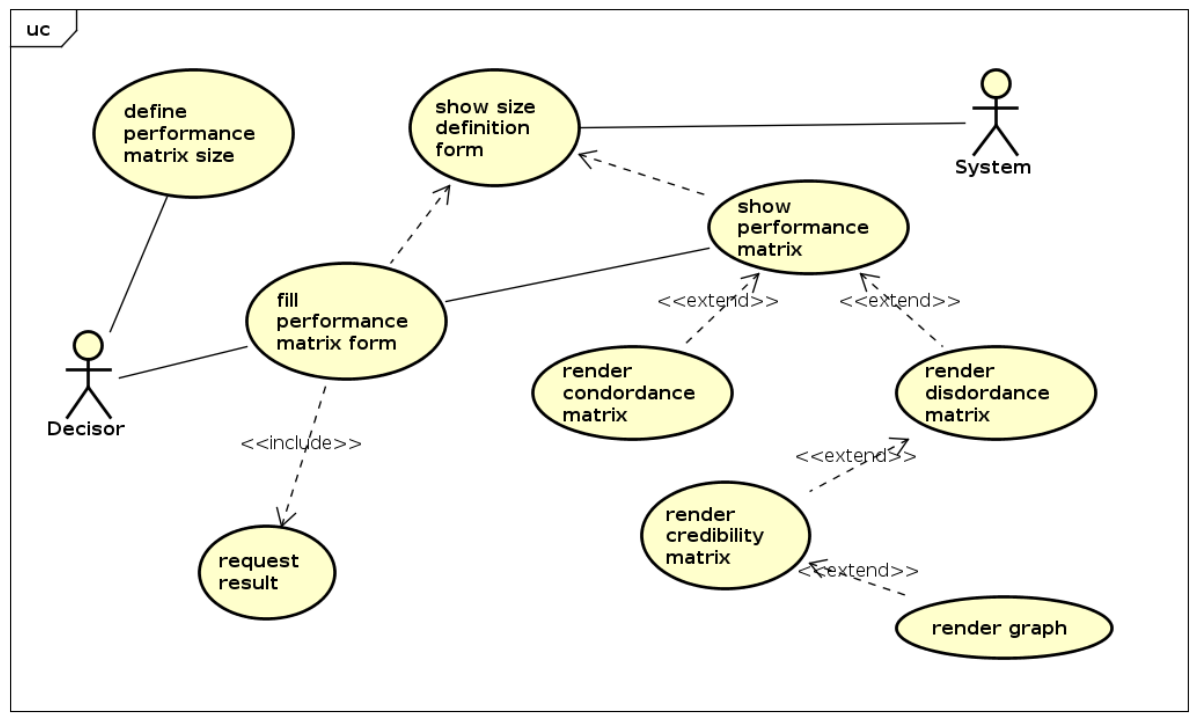

\subsection{IMPLEMENTAÇÃO}

Os scripts foram empacotados na estrutura MVC da seguinte forma:

- Projeto JSEletcre v1.0

- Documentação e modelagem

- UML

- Planilha exemplo

- Diagrama

- Artigo / manual

- controle

- Coleção de funções com as regras de negócio (JavaScript)

$\checkmark \underline{\text { Visão }}$

- Pacote da biblioteca gráfica (JavaScript)

- Pacote de estilos visuais e design (CSS)

- $\quad$ Pacotes de imagens (png, jpg, gif, bmp)

- Biblioteca Jquery (JavaScript)

- Script de interface entre a controladora e a visão (JavaScript)

- Página principal (HTML) 
Figura 3: Um dos métodos da controladora. Fonte: Autores (2019)

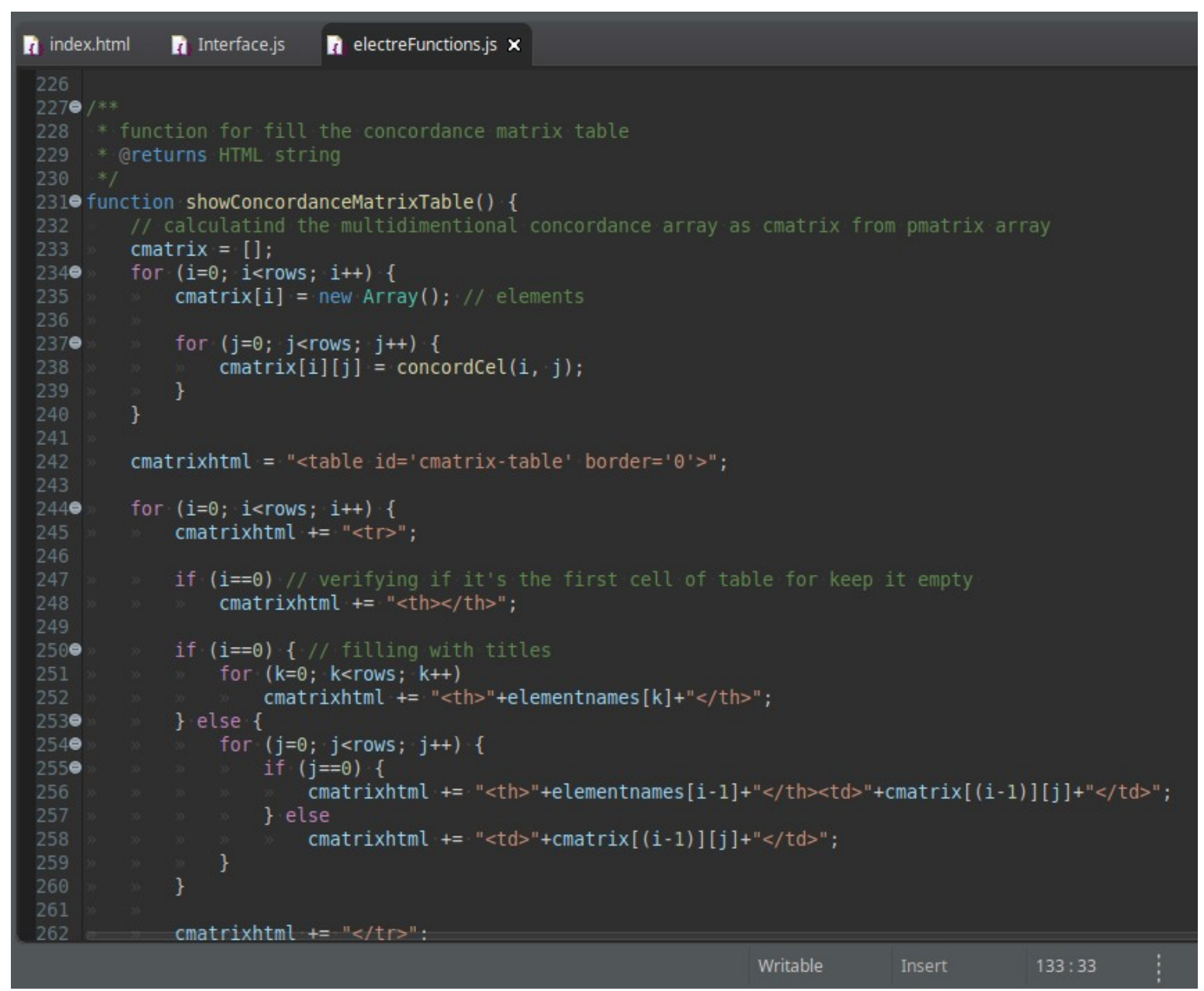

\section{APLICAÇÃO}

A título de teste e validação da ferramenta JSElectre v1.0, foi considerada uma situação hipotética em que cinco professores de um determinado colégio foram avaliados segundo cinco critérios, conforme a Tabela 6. 
Tabela 6: Matriz de performance hipotética. Fonte: Autores (2019)

\begin{tabular}{|c|c|c|c|c|c|}
\hline Critérios & $\begin{array}{c}\text { Experiência } \\
\text { Profissional }\end{array}$ & Carisma & $\begin{array}{c}\text { Conhecimen } \\
\text { to Técnico }\end{array}$ & $\begin{array}{c}\text { Expressão } \\
\text { Escrita }\end{array}$ & $\begin{array}{c}\text { Expressão } \\
\text { Oral }\end{array}$ \\
\cline { 1 - 5 } Professores & 2 & 8 & 4 & 4 & 4 \\
\hline Alice & 5 & 7 & 7 & 7 & 7 \\
\hline Bruna & 3 & 2 & 1 & 2 & 2 \\
\hline Carlos & 1 & 2 & 3 & 1 & 1 \\
\hline Daniel & 7 & 6 & 5 & 5 & 5 \\
\hline Ester & $\mathbf{2}$ & $\mathbf{1}$ & $\mathbf{3}$ & $\mathbf{2}$ & $\mathbf{2}$ \\
\hline Peso dos & & & & & \\
\hline
\end{tabular}

A partir desta avaliação, supõe-se que o colégio decide utilizar o método ELECTRE I para selecionar a melhor equipe para representar a instituição em uma competição escolar entre professores.

A interface gráfica do JSElectre v1.0 está projetada para executar o método ELECTRE I, de maneira que são necessários somente três passos para alcançar a completa resolução do problema.

O primeiro passo já é exibido na página inicial da aplicação como demonstrado na Figura 4, onde no quadrante size of matrix, deverá ser informada a quantidade de linhas (critérios) e a quantidade de colunas (elementos) para que em seguida, após o evento de clique no botão new, o JSElectre possa renderizar dinamicamente um novo formulário para preenchimento da matriz de performance no tamanho informado. 
Figura 4: Tela inicial do JSElectre v1.0. Fonte: Autores (2019)

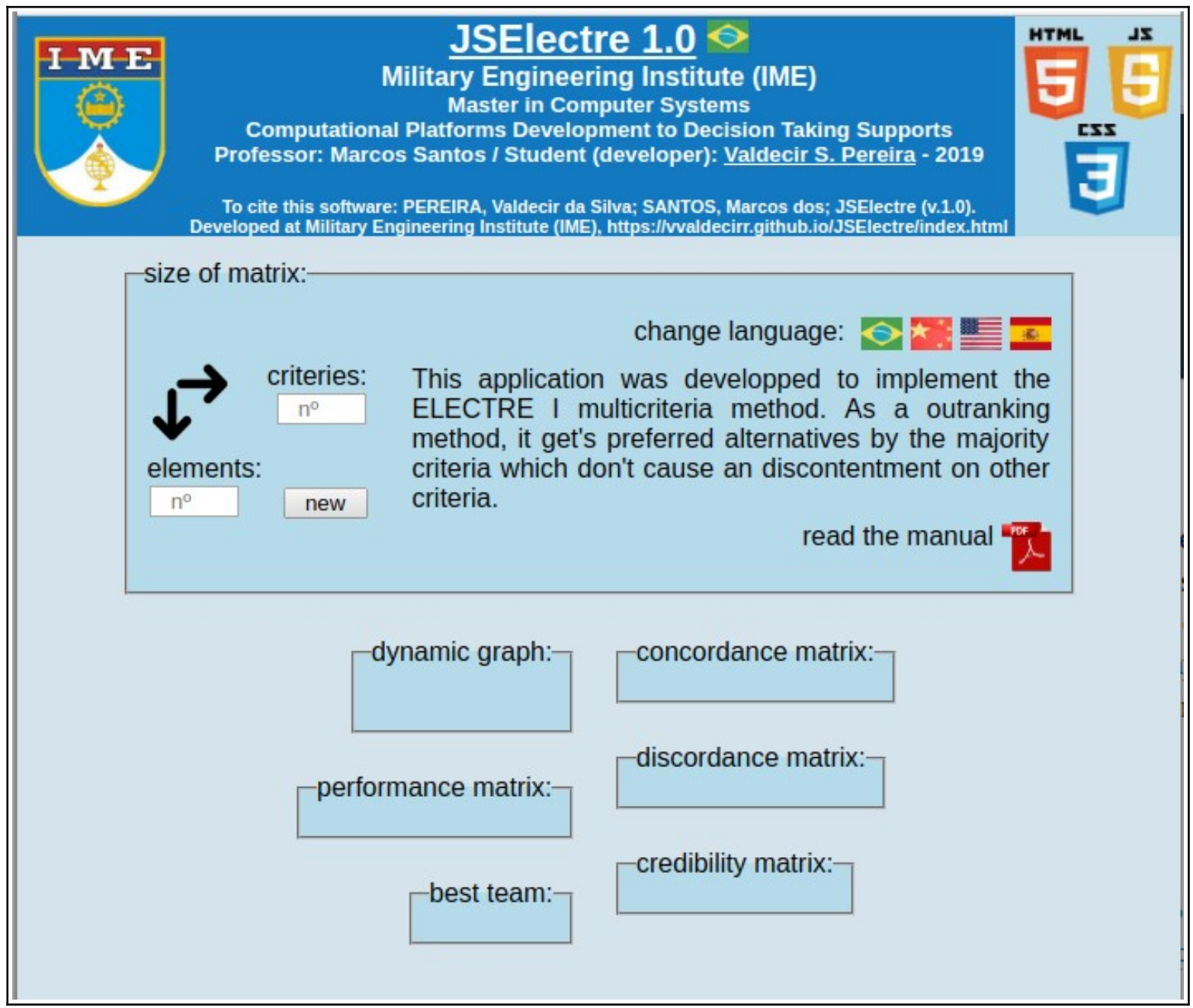

Uma vez já renderizado o formulário da matriz de performance no quadrante performance matrix, como exibido na Figura 5, no segundo passo basta preencher com os valores reais definidos para cada critério referente aos seus respectivos elementos (no exemplo definidos como professores), preencher os índices de importância dos pesos de cada critério (respeitando o somatório que deverá ser no valor de 10) e definir os índices de sobreclassificação para concordância e para discordância. Por padrão a aplicação já renderiza o formulário com os índices na atribuição mais rigorosa.

Todos os campos numéricos são incrementáveis e decrementáveis por meio do clique do mouse, setas direcionais para cima e para baixo no teclado ou a forma tradicional digitada. 
Figura 5: Preenchimento da matriz de performance. Fonte: Autores (2019)

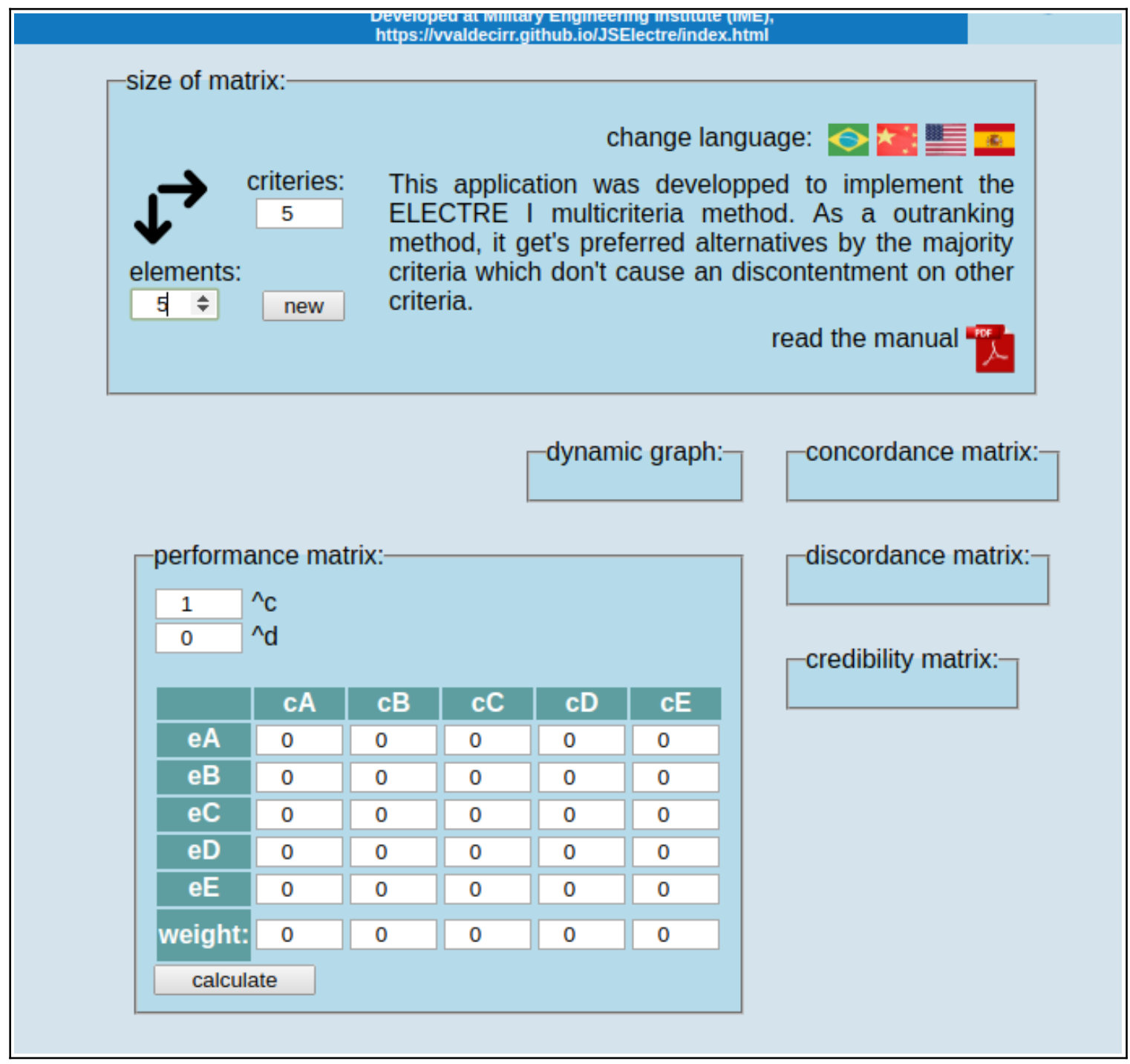

Após o completo preenchimento dos campos do formulário, no terceiro passo deverse-á executar a ação de clique no botão calculate, e o sistema executará o algoritmo do método sob os dados informados renderizando em seguida as matrizes de concordância, discordância, credibilidade e o grafo de vértices auto ajustável espacialmente nos seus respectivos quadrantes como demonstra a Figura 6 e a rotina está completa. 
Figura 6: Matrizes de concordância, discordância, credibilidade e grafo. Fonte: Autores (2019)

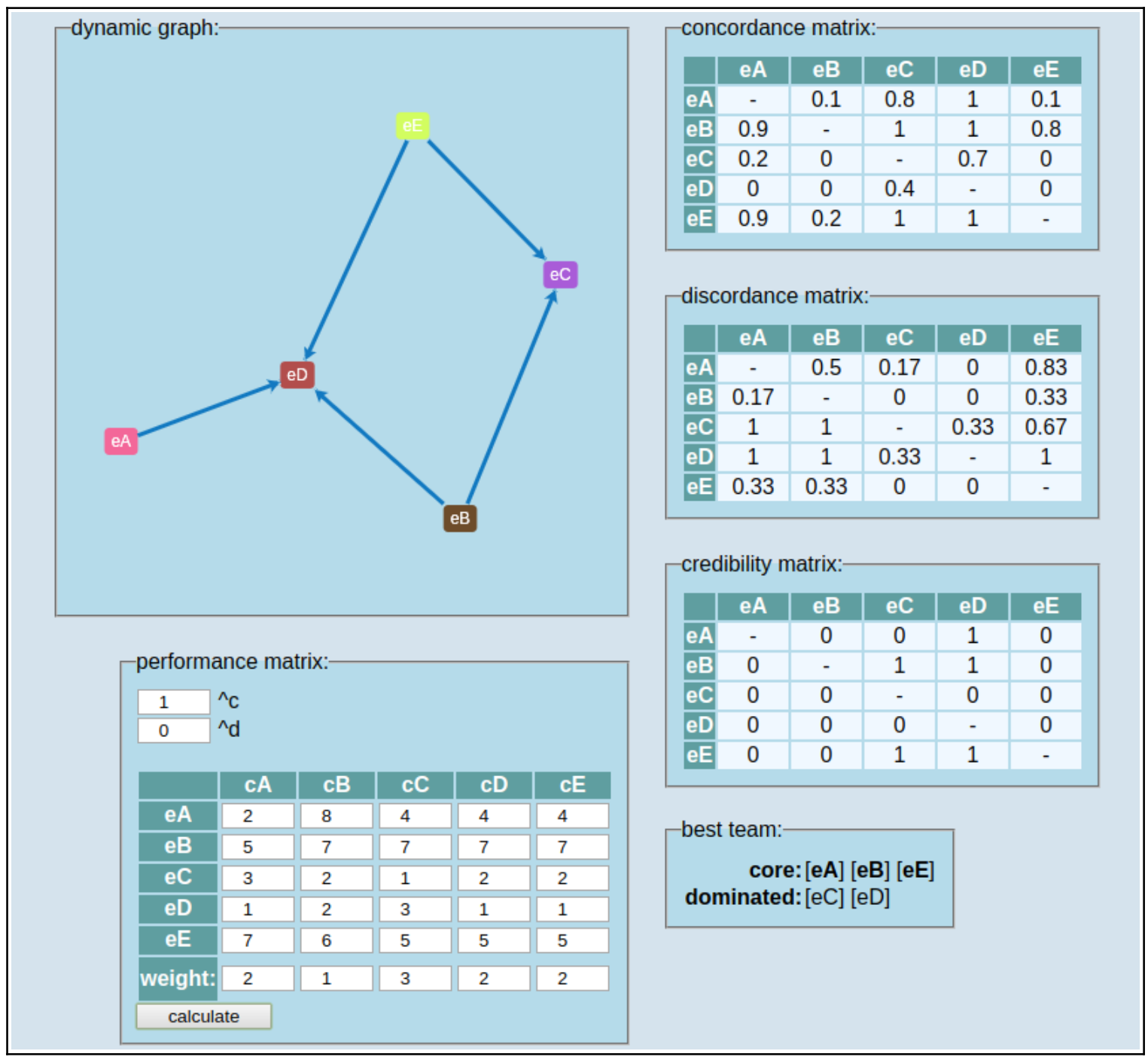

\section{CONTRIBUIÇÕES DA FERRAMENTA}

Como a aplicação JSElectre foi desenvolvida especialmente no modelo client side (execução do lado do cliente), todo o algoritmo é interpretado e executado pelo navegador de páginas de internet utilizado no terminal do usuário, dispensando assim a necessidade do usuário possuir algum conhecimento técnico prévio. Acredita-se ainda no elevado poder de melhoria da ferramenta, uma vez que, por ter sido desenvolvida em uma das linguagens de programação mais populares entre os programadores front-end (JavaScript), qualquer desenvolvedor pode requerer a submissão de seus próprios códigos-fonte online como contribuição às melhorias funcionais.

\section{TRABALHOS FUTUROS}

Como melhorias pretendidas e já escalonadas ao escopo do software JSElectre, abaixo estão listados os seguintes recursos a serem implementadas nas versões seguintes:

Internacionalização: Refere-se à disponibilização da tradução de todos os elementos textuais componentes da página da aplicação para a maioria dos idiomas possíveis visando assim uma difusão internacional e inteligível à comunidade global. 
Índices de peso: Consistirá em informar automaticamente ao usuário da aplicação, se o total de valor ideal para o cálculo dos índices de peso estão dentro da faixa esperada obedecendo assim fielmente as regras da metodologia impedindo um somatório do peso e os restantes dos cálculos inválidos por desatenção do usuário.

Relações de preferência: Para que a ferramenta pudesse ser imediatamente disponibilizada para a comunidade, nesta primeira versão do JSElectre, optou-se por utilizar somente a relação de preferência do Critério Verdadeiro. No entanto, pretende-se implementar as relações restantes da metodologia Semi-Critério e Pseudo-Critério.

Grafo: Após uma futura discussão do quanto possa evoluir sem descaracterizar a metodologia raiz do ELECTRE I, deseja-se incrementar o grafo resultante, destacando os vértices detectados como dominantes sob renderização com um tamanho ligeiramente, mas perceptível, maior do que os vértices sobrepostos, visando enriquecer a qualidade das informações possíveis de serem captadas somente com a visualização do grafo.

Web service: Com o intuito de seguir a boa prática do baixo acoplamento e alta coesão com escalabilidade operacional do código fonte da ferramenta, pretende-se refatorar toda a estrutura da aplicação para que também passe a prover um serviço de consulta web service REST, permitindo a reutilização dos métodos internos do JSElectre por aplicativos de terceitos que poderão usufruir diretamente do retorno dos resultados em seus programas.

Versões do electre: Visando consolidação e validação da relevância do JSElectre na comunidade, pretende-se implementar na ferramenta a abrangência das versões II, III, IV e TRI da metodologia ELECTRE original.

Nomeação de critérios e elementos: Acredita-se que, apesar de dispensável para alguns usuários, será interessante possibilitar que o usuário, caso deseja, renomeie os critérios e elementos que são atribuídos automaticamente pelo sistema com as letras do alfabeto.

Normalização: O intuito é viabilizar a flexibilidade de inversão da proporcionalidade do critério quando estes ainda não estiverem normalizados, compatibilizando também assim com eventos de critérios inversamente proporcionais aos outros.

\section{REFERÊNCIAS BIBLIOGRÁFICAS}

[1] MARLINDA, Linda; BAIDAWI, Taufik; DURACHMAN, Yusuf; AKMALUDIN, Akmaludin, at al. A Multi-Study Program Recommender System Using ELECTRE Multicriteria Method. IEEE 5th International Conference on Cyber and IT Service Management (CITSM), 2017.

[2] MARTIN, Kannan Govindan; JEPSEN, Brandt, in. ELECTRE: A comprehensive literature review on methodologies and applications. European Journal of Operational Research, abr. 2016.

[3] PEREIRA, Valdecy, in. MCDA-01-ELECTRE I: Auxílio Multicritério à Decisão. Universidade Federal Fluminence, 2017. Disponível em: https:/www.researchgate.net/publication/319504754_MCDA-01-ELECTRE_I, set. 2017.

[4] EDUARDO. O que é o HTML5. DEVMEDIA, 2012. Disponível em: https://www.devmedia.com.br/o-que-e-o-html5/25820. Acesso em: 27 ago. 2019.

[5] ARRIGONI, Ricardo. Introdução às novidades do CSS3. LINHA DE CÓDIGO. Disponível em: http://www.linhadecodigo.com.br/artigo/3566/introducao-as-novidades-do-css3.aspx. Acesso em: 27 ago. 2019. 
[6] BIZZO, Mateus, at al. O que é JavaScript?. Mozilla.org, 2019. Disponível em: https://developer.mozilla.org/pt-BR/docs/Learn/JavaScript/First_steps/O_que_e_JavaScript. Acesso em: 27 ago. 2019.

[7] TEIXEIRA, José Riacardo. O Que é jQuery e Para Que Serve?. DEVMEDIA, 2013. Disponível em: https://www.devmedia.com.br/jquery-tutorial/27299. Acesso em: 27 ago. 2019.

[8] CLÍMACO, João; DIAS, Luís; ALLMEIDA, Luís Alçada, in. Uma revisão de três pacotes de software dedicados a problemas multiatributo. ENGEVISTA, V. 17, n. 3, p. 288-307, set. 2015.

[9] ARAUJO, Jefferson de Jesus; AMARAL, Thiago Magalhães, in. Aplicação do método ELECTRE I para problemas de seleção envolvendo projetos de desenvolvimento de software livre. Gestão da Produção, Operações e Sistemas, Bauru, Ano 11, n 2, abr-jun/2016, p. 121-137. 